\title{
Double Adaptive Filtering of Gaussian Noise Degraded Images
}

\author{
Tuan D. Pham ${ }^{1,2}$ \\ ${ }^{1}$ Bioinformatics Applications Research Centre \\ 2 School of Mathematics, Physics, and Information Technology \\ James Cook University \\ Townsville, QLD 4811, Australia \\ tuan.pham@jcu.edu.au
}

\begin{abstract}
Good estimate and simulation of the behavior of additive noise is central to the adaptive restoration of images corrupted with Gaussian noise. This paper presents a double adaptive filtering scheme in the sense that the filter is able to estimate the variance of additive noise in order to determine the filter gain for pixel updating, and also able to decide if the pixel should remain unfiltered. Experimental results obtained from the restoration of several images have shown the superiority of the proposed method to some benchmark image filters.
\end{abstract}

Keywords: Image restoration, Gaussian noise, adaptive filtering.

\section{Introduction}

The topic of restoring noisy images to its original verions is one of important research areas in image processing and computer vision. An optimal image restoration is usually based on some criteria for noise modeling and noise deduction. There are several types of noise that can degrade orginal images such as Gaussian (additive), Poisson, salt \& pepper (on and off), and speckle (multiplicative). Thus, there are many methods proposed for dealing with different types of noise in images. Literature review in image restoration is huge but most methods are based on the methodologies of linear, non-linear, and adaptive filtering [5]. However, the problem of image restoration still remains challengingly open because image restoration is difficult since it is an ill-posed inverse problem - necessary information required about the degraded image to reconstruct the original is inherently imprecise. Therefore, methods for effective and better solutions continue to be developed.

Awate and Whitaker [1] have recently proposed an adaptive image filtering scheme for restoring degraded images by comparing pixel intensities having similar neighborhood in the image. Although this work makes no assumption about the properties of image signal and noise, it is empirically observed to perform best with additive noise. Other recently developed methods for image restoration are wavelet-based image denoising [10, mean-shift algorithms [2, and iterative function [16], pointspread function [3], and histogram-based fuzzy filter [15]. In 
particular, for additive noise, the principle of Wiener filtering is still one of the earliest and best known approaches for adaptive image filtering [5]. Based on this motivation, our contribution focuses on the reliable estimate of noise which is normally assumed in the Wiener filter process. We then extend the algorithm with an ability to detect noisy pixels to be adaptively updated. The rest of this paper is organized as follows. To facilitate the discussion of the new algorithms, basic formulation of adaptive Wiener filtering is outlined in Section 2. Section 3 presents a geostatistics-based method for noise estimation. In Section 4, the concept of spatial correlation in geostatistics is utilized to derive a decision rule for noise detection. Section 5 illustrates the performance of the proposed method and comparisons. In Section 6, we conclude our contribution and suggest further development of the proposed method.

\section{Basic Adaptive Restoration Process}

Let $f(x, y)$ be the ideal image, $g(x, y)$ the degraded image, and $v(x, y)$ the white noise which is additive, stationary, and has zero mean. The degraded image can be modeled as

$$
g(x, y)=f(x, y)+v(x, y)
$$

Given the observed image $g(x, y)$ and the noise statistics, the task of image restoration is to recover the signal as close as possible to the ideal image $f(x, y)$. Space-variant Wiener filters have been designed for such task, and a specific algorithm can be described as follows [9].

The power spectrum of the noise $v(x, y)$ with variance $\sigma_{v}^{2}$ is given by

$$
P_{v}\left(\omega_{x}, \omega_{y}\right)=\sigma_{v}^{2}
$$

Assuming that $f(x, y)$ is stationary within a small local image $\mathcal{L}$, then $f(x, y)$ can be modeled by its local mean $m_{f}$, local standard deviation $\sigma_{f}$ and the white noise $n(x, y)$ that has zero mean and unit variance:

$$
f(x, y)=m_{f}+\sigma_{f} n(x, y)
$$

The Wiener filter whose frequency reponse $H\left(\omega_{x}, \omega_{y}\right)$ within $\mathcal{L}$ is

$$
\begin{aligned}
H\left(\omega_{x}, \omega_{y}\right) & =\frac{P_{f}\left(\omega_{x}, \omega_{y}\right)}{P_{f}\left(\omega_{x}, \omega_{y}\right)+P_{v}\left(\omega_{x}, \omega_{y}\right)} \\
& =\frac{\sigma_{f}^{2}}{\sigma_{f}^{2}+\sigma_{v}^{2}}
\end{aligned}
$$

From (5), a scaled impulse response $h(x, y)$ is given as

$$
h(x, y)=\frac{\sigma_{f}^{2}}{\sigma_{f}^{2}+\sigma_{v}^{2}} \delta(x, y)
$$


From (5), the restored image $r(x, y) \in \mathcal{L}$ can be obtained by

$$
\begin{aligned}
r(x, y) & =m_{f}+\left[g(x, y)-m_{f}\right] * \frac{\sigma_{f}^{2}}{\sigma_{f}^{2}+\sigma_{v}^{2}} \delta(x, y) \\
& =m_{f}+\frac{\sigma_{f}^{2}}{\sigma_{f}^{2}+\sigma_{v}^{2}}\left[g(x, y)-m_{f}\right]
\end{aligned}
$$

in which $*$ denotes the convolution operator.

If the local mean and standard deviation are updated at each pixel, we have 7

$$
r(x, y)=m_{f}(x, y)+k(x, y)\left[g(x, y)-m_{f}(x, y)\right]
$$

where $k(x, y)$ is a filter gain and defined as

$$
k(x, y)=\frac{\sigma_{f}^{2}(x, y)}{\sigma_{f}^{2}(x, y)+\sigma_{v}^{2}}
$$

The local mean and local standard deviation described in (9) can be estimated using local statistics [7]

$$
m_{f}(x, y)=\frac{1}{N M} \sum_{k, l \in w} g(k, l)
$$

where $w$ is a local $N \times M$ window of the image.

And

$$
\sigma_{f}^{2}(x, y)=\frac{1}{N M} \sum_{k, l \in w}\left[g(k, l)-m_{f}(x, y)\right]^{2}
$$

If the noise variance is not known then it can be approximated as the mean of all the local variances in the image $I$, i.e.,

$$
\sigma_{v}^{2} \approx \frac{1}{N M} \sum_{k, l \in I} \sigma_{f}^{2}(k, l)
$$

We are particularly interested in estimating the variance of white additive noise in images using the variogram function introduced in geostatistics, which models the spatial distribution of an image with a random function and estimates the noise variance as the expectation of the squared error of the random variables separated by a spatial distance. We then seek to formulate a rule to decide if a pixel value being corrupted with noise. This decision is based on the comparison of the long-range and local spatial probabilities of a pixel intensity. 


\section{Estimating Additive Noise with Geostatistics}

The theory of geostatistics is based on the notion of regionalized variables and their random functions [6]. A variable $z(p)$ that is distributed in space $\Omega$ is said to be regionalized and represented as a realization of a random function $Z(p)$ :

$$
Z(p)=\left\{Z\left(p_{i}\right), \forall p_{i} \in \Omega\right\}
$$

Let $z(p)$ and $z(p+h)$ be two real values at two locations $p$ and $p+h$. The spatial variance of these two points is expressed by the variogram function $2 \gamma(p, h)$, which is defined as the expectation of the squared difference of the two random variables

$$
2 \gamma(p, h)=E\left\{[Z(p)-Z(p+h)]^{2}\right\}
$$

The estimation of (14) requires several realizations of the pair of random variables $[Z(p)-Z(p+h)]$, i.e., $\left[z_{1}(p), z_{1}(p+h)\right],\left[z_{2}(p), z_{2}(p+h)\right], \ldots,\left[z_{k}(p), z_{k}(p+h)\right]$. However, in many applications, only one realization $[z(p), z(p+h)]$ can be available, that is the actual measure of the values at point $p$ and $p+h$. To overcome this problem, the intrinsic hypothesis [6] is introduced, which states that a random function $Z(p)$ is intrinsic when

- its mathematical expectation exists and does not depend on $p$ :

$$
E\{Z(p)]\}=m, \forall p
$$

- the increment $[Z(p)-Z(p+h)]$ has a finite variance which does not depend on $p$ :

$$
\operatorname{Var}\{Z(p)-Z(p+h)\}=E\left\{[Z(p)-Z(p+h)]^{2}\right\}=2 \gamma(h), \forall p
$$

The variogram $2 \gamma(h)$ is therefore constructed using the actual data as follows.

$$
2 \gamma(h)=\frac{1}{N(h)} \sum_{i=1}^{N(h)}\left[z\left(p_{i}\right)-z\left(p_{i}+h\right)\right]^{2}
$$

where $N(h)$ is the number of experimental pairs $\left[z\left(p_{i}\right)-z\left(p_{i}+h\right)\right]$ of the data separated by $h$.

As all the random variables of the random function have the same mean and variance, expanding (15) we have:

$$
\begin{aligned}
\gamma(h) & =\frac{1}{2} E\left\{[Z(p+h)-Z(p)]^{2}\right\} \\
& =\frac{1}{2} E\left\{Z(p+h)^{2}\right\}+\frac{1}{2} E\left\{Z(p)^{2}\right\}-E\{Z(p+h) Z(p)\} \\
& =E\left\{Z^{2}\right\}-E\{Z(p+h) Z(p)\} \\
& =E\left\{Z^{2}\right\}-m^{2}-\left[E\{Z(p+h) Z(p)\}-m^{2}\right] \\
& =\sigma^{2}-\operatorname{Cov}\{Z(p+h) Z(p)\}
\end{aligned}
$$


Based on (13), (14), and (19), we can draw two conceptual aspects as follows.

1. The variogram can be viewed as an estimation variance in which an error occurs when the value at $p$ is estimated by the value at $p+h$, i.e.,

$$
\sigma_{E}^{2}=E\left\{[Z(p)-Z(p+h)]^{2}\right\}
$$

2. Given that the location $p+h$ is sufficiently close to $p$, then $z(p+h)$ can be considered to be another realization of $Z(p)$, i.e., $z(p+h) \approx z_{k}(p)$.

We therefore can approximate the variance of additive noise in an image as the variogram where $h=1$ expressed in pixels:

$$
\sigma_{v}^{2} \approx \sigma_{E}^{2}=2 \gamma(h=1)
$$

It is realized that we can deduce to estimate the noise variance in an image from the experimental variogram at $h=0$. Such estimation can be obtained by extrapolation [6], and has been applied for calculating the standard deviations of non-uniform images [12]. However, extrapolation is less accurate and the extrapolation proposed in [12] is not suitable for automatic processing.

\section{Detection of Noisy Pixels}

Let $I$ be an image, and let $f_{i}$ and $f_{j}$ be the gray levels of pixels located at positions $i$ and $j$ in $I$ respectively. The purpose is to determine the probability of the spatial non-randomness of the pixel considered for filtering within a neighborhood. This probability is called the local probability of spatial nonrandomness. If this probability is higher than that of the long-range probability of non-randomness of the same pixel intensity, then its intensity value is considered as uncorrupted and therefore remains unchanged; otherwise the pixel will be filtered. We proceed the determination of these two types of probability as follows.

Let $T$ be the gray-level threshold of $I$. The thresholded image of $I$ at $T$ is defined as

$$
y_{i}(T)=\left\{\begin{array}{lll}
1 & : & f_{i}=T \\
0 & : & f_{i} \neq T
\end{array}\right.
$$

Utilizating the concepts of variograms and histograms, the long-range probability of spatial non-randomness of pixels having intensity value $T$ separated by lag distance $h$ over $I$, denoted as $P_{G}(T, h)$, can be defined by

$$
P_{G}(T, h)=\frac{1}{N(h)} \sum_{(i, j) \in I \mid h_{i j}=h} \delta_{i j}(h)
$$


where $N(h)$ is the total number of pairs of pixels in $I$ separated by lag distance $h, h_{i j}$ is the distance between $f_{i}$ and $f_{j}$, and $\delta_{i j}(h)$ behaves like a Kronecker delta which is defined as

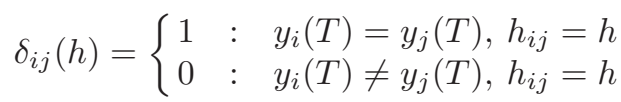

Similarly, the local probability of the spatial non-randomness of pixels having intensity value $T$ separated by lag distance $h$ over some chosen window $w_{L}$, denoted as $P_{L}(T, h)$, can be defined by

$$
P_{L}(T, h)=\frac{1}{M(h)} \sum_{(i, j) \in w_{L} \mid h_{i j}=h} \delta_{i j}(h)
$$

where $M(h)$ is the total number of pairs of pixels in window $w_{L}$ separated by lag distance $h$.

It is known that the variogram is very useful for exploring the features of spatial data, and can help to detect anomalies, inhomogeneities and randomness of sample values in spatial domain [14. We use the conceptual framework of calculating the variogram and the histogram to compute the probability that indicates the chance of a pixel intensity being located closely to each other in space. We then make an assumption that if the local probability exceeds the global (long-range) probability, then the pixel is not corrupted with noise. Thus, the decision to either retain or update each pixel is according to the following rules:

$$
\begin{array}{ll}
P_{L}(T, h)>P_{G}(T, h) & : \quad \text { no updating } \\
P_{L}(T, h) \leq P_{G}(T, h) & : \quad \text { updating }
\end{array}
$$

\section{Experiments}

We test the algorithm with several images as shown in Figure 1 which consists of 4 different original images. Those images are selected based on the good content of different details, texture, regions, and shading. To measure the performance of the restoration, the signal-to-noise ratio (SNR) improvement expressed in $\mathrm{dB}$ is used, which is defined by 9 ]

$$
\text { SNR Improvement }=10 \log _{10} \frac{\operatorname{NMSE}(f, g)}{\operatorname{NMSE}(f, r)}
$$

where the normalized mean square error (NMSE) between the original image $f$ and the restored image $r$ is defined by

$$
\operatorname{NMSE}(f, r)=100 \frac{\operatorname{Var}(f-r)}{\operatorname{Var}(f)} \%
$$

All images were processed with a $3 \times 3$ filter window $(w)$ which was suggested by [7] as a fairly good choice. To compute the local probability, we set the size 

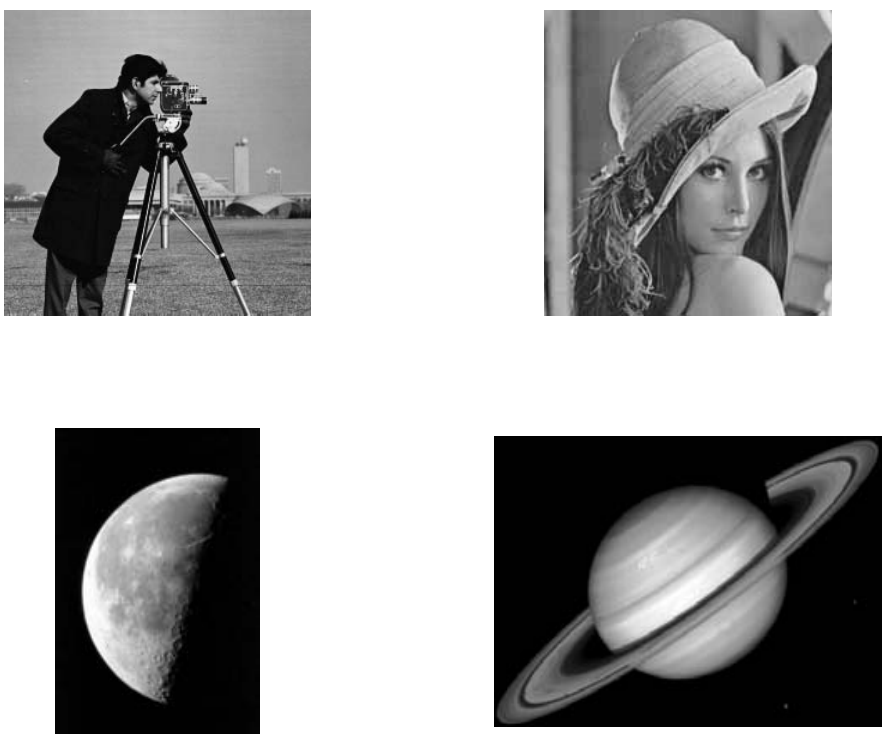

Fig. 1. Original images: Top left: photographer, top right: Lenna, bottom left: moon, bottom right: saturn
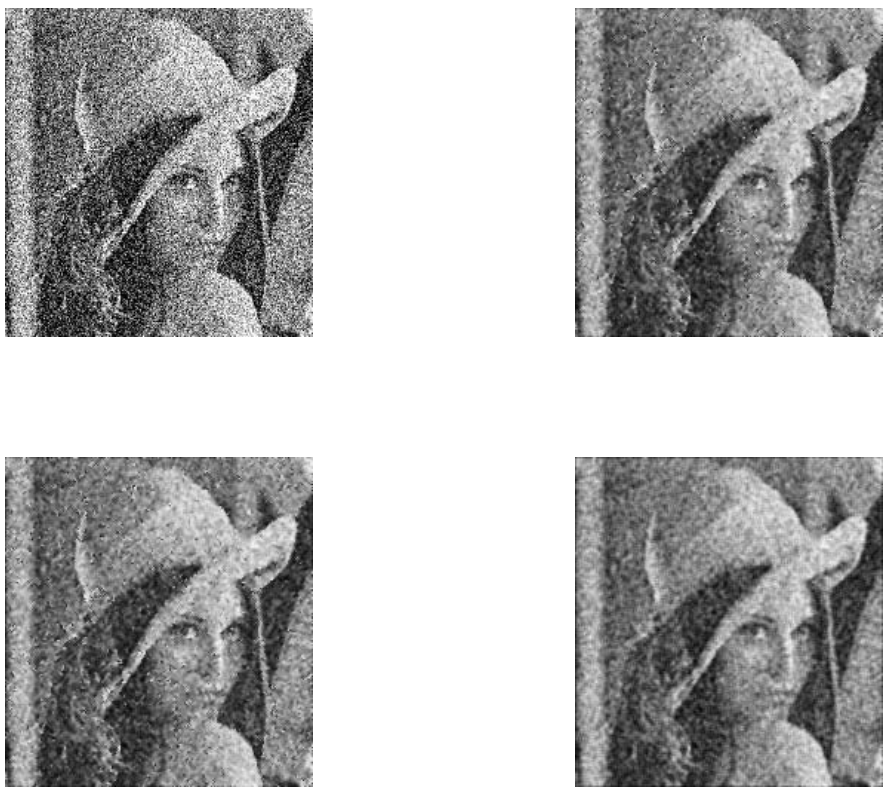

Fig. 2. Top left: degraded image with Gaussian noise (0 mean, 0.05 variance), top right: adaptive Wiener filter, bottom left: mean filter, bottom right: double adaptive filter 

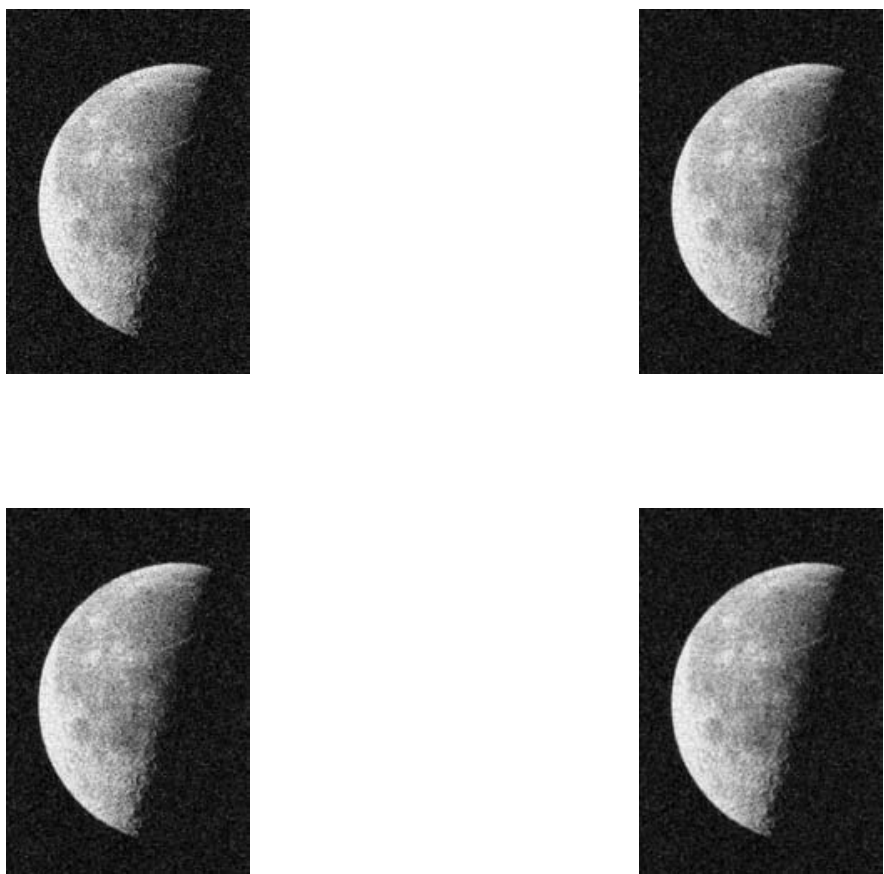

Fig. 3. Top left: degraded image with Gaussian noise (0 mean, 0.05 variance), top right: adaptive Wiener filter, bottom left: mean filter, bottom right: double adaptive filter

of the local window $\left(w_{L}\right)$ to be $9 \times 9$ which is considered sufficient enough to calculate the spatial correlation (in geostatistics, the number of data points being greater than 25 can be considered sufficent to compute the semi-variogram). The original images were corrupted with zero-mean Gaussian white noise of variances of 0.02 and 0.05 respectively. Tables 1 and 2 show the SNR improvements of the processed images obtained by the adaptive Wiener filter method, the mean filter, and the proposed method where single adaptive does not employ the noise detection procedure but the double adaptive does. The proposed method gives the highest SNR improvements in all cases; whereas and the adaptive Wiener filter gives higher SNR improvements than the mean filter. Figure 2 shows the moon image degraded with zero-mean Gaussian noise of variances of 0.05 and its restored versions obtained from the three methods. In addition to its highest SNR, the restored image achieved from the proposed filter can be observed that noise is suppressed with smoother effect than the average filter, and the edges can still be better reserved than the adaptive Wiener filter.

To compare with other recently developed methods, we used the Lenna image whose size is larger than that used in UNITA method developed by Awate and 
Table 1. SNR Improvements (dB) - Gaussian Noise (zero mean, 0.02 variance)

\begin{tabular}{|c|c|c|c|c|}
\hline Image & \multicolumn{4}{|c|}{ Adaptive Wiener Mean Single Adaptive Double Adaptive } \\
\hline Lena $(222 \times 208)$ & 6.86 & 6.46 & 7.56 & 8.23 \\
\hline photographer $(256 \times 256)$ & 6.16 & 5.89 & 6.31 & 7.45 \\
\hline moon $(900 \times 1200)$ & 5.30 & 5.16 & 7.16 & 8.01 \\
\hline saturn $(900 \times 1201)$ & 5.09 & 4.98 & 7.13 & 8.46 \\
\hline Logo database & 5.13 & 4.64 & 5.29 & 6.77 \\
\hline
\end{tabular}

Table 2. SNR Improvements (dB) - Gaussian Noise (zero mean, 0.05 variance)

\begin{tabular}{lcccc}
\hline Image & Adaptive Wiener Mean & Single Adaptive Double Adaptive \\
\hline Lena $(222 \times 208)$ & 6.96 & 6.76 & 8.17 & 9.82 \\
photographer $(256 \times 256)$ & 6.27 & 6.09 & 6.99 & 8.01 \\
moon $(900 \times 1200)$ & 5.24 & 5.18 & 7.11 & 9.14 \\
saturn $(900 \times 1201)$ & 5.16 & 5.12 & 7.17 & 9.10 \\
Logo database & 5.24 & 4.78 & 5.92 & 7.24 \\
\hline
\end{tabular}

Table 3. RMSE ratios of different methods using Lenna image

\begin{tabular}{lllll}
\hline UNITA & WF1 & WF2 & WF3 & DA \\
\hline 0.46 & 0.36 & 0.38 & 0.41 & 0.38 \\
\hline
\end{tabular}

Whitaker 1 . The image was simulated with the same level of the additive noise to carry out the restoration using the proposed double adaptive filter (denoted as DA). We then used the root-mean-square error (RMSE) ratio of the initial and restored RMSE errors to compare the results obtained from the UNITA, waveletbased filter (denoted as WF1) developed by Portilla et al. [11, wavelet-based filter (denoted as WF2) developed by Sender and Selesnick [13, and waveletbased filter (denoted as WF3) developed by Pizurica et al. [10. The results from these three wavelet-based methods were taken from [1. Table 3 show the error ratios obtained from the five models. The error ratio of the proposed method is equivalent to that of WF2, higher than WF1, and lower than UNITA and WF3. However, it was mentioned in [1] that although the wavelet-based approach gave lower error than that of the UNITA, it has ringing-like artifacts in smooth regions. Our proposed method yields lower error ratio than the UNITA filter and still does not suffer from that effect. We further tested the same image restoration methods using the logo database of the University of Maryland [4] that consists of 105 intensity logo images of scanned binary versions. The results obtained show similar analysis addressed earlier, where the proposed double filtering approach gives the best improvements. 


\section{Conclusions}

The ideas of spatial relation and spatial randomness modeled by the theory of regionalized variables and geostatistics have been utilized to largely improve the well-known principle of adaptive Wiener filter for restoring images degraded with Guassian white noise. The experiments have shown that this algorithm provided the most favorable results by means of the SNR improvements and visual observation over some benchmark methods. The proposed method appears to be better or competitive with some other recently developed techniques. In addition, the computational procedure and speed of the proposed method is very easy for computer implementation, and can be extended for filtering other types of noise in images.

\section{References}

1. Awate, S.P., Whitaker, R.T.: Unsupervised, information-theoretic, adaptive image filtering for image restoration. IEEE Trans. PAMI 28, 364-376 (2006)

2. Barash, D., Comaniciu, D.: A Common Framework for Nonlinear Diffusion, Adaptive Smoothing, Bilateral Filtering and Mean Shift. Image Vision Computing 22, 73-81 (2004)

3. Ferreira, V.C., Mascarenhas, N.D.A.: Analysis of the Robustness of Iterative Restoration Methods with Respect to Variations of the Point Spread Function. In: Proc. ICIP'00 III, 789-792 (2000)

4. ftp://ftp.cfar.umd.edu/pub/documents/contrib/database/UMDlogo_ database.tar

5. Gonzalez, R.C., Woods, R.E., Eddins, S.L.: Digital Image Processing using Matlab. Pearson Prentice Hall, Englewood Cliffs (2004)

6. Journel, A.G, Huijbregts, C.J.: Mining Geostatistics. Academic Press, Chicago (1978)

7. Lee, J.-S.: IEEE Trans. PAMI, vol. 2 pp.165-168 (1980)

8. Lee, J.-S., Hoppel, K.: Noise modeling and estimation of remotely-sensed images. In: Proc. Int. Conf. Geoscience and Remote Sensing 2, 1005-1008 (1989)

9. Lim, J.S.: Two-Dimensional Signal and Image Processing. Prentice-Hall, Englewood Cliffs (1990)

10. Pizurica, A., Philips, W., Lemahieu, I., Acheroy, M.: A joint inter and intrascale statistical model for Bayesian wavelet based image denoising. IEEE Trans. Image Processing 11, 545-557 (2002)

11. Portilla, J., Strela, V., Wainwright, M., Simoncelli, E.: Image denoising using scale mixtures of Gaussians in the wavelet domain. IEEE Trans. Image Processing 12, 1338-1351 (2003)

12. Sanchez-Brea, L.M., Bernabeu, E.: On the standard deviation in charge-coupled device cameras: A variogram-based technique for nonuniform images. J. Electronic Imaging 11, 121-126 (2002)

13. Sendur, L., Selesnick, I.: Bivariate shrinkage functions for wavelet-based denoising exploiting interscale dependency. IEEE Trans. SP 50, 2744-2756 (2002)

14. Wackernagel, H.: Multivariate Geostatistics. Springer, Heidelberg (2003)

15. Wang, J.H., Liu, W.J., Lin, L.D.: Histogram-based fuzzy filter for image restoration. IEEE Trans. SMC - Part B: Cybernetics 32, 230-238 (2002)

16. Xue, F., Liu, Q.S., Fan, W.H.: Iterative Image Restoration using a Non-Local Regularization Function and a Local Regularization Operator. In: Proc. ICPR'06 III, 766-769 (2006) 